Check for updates

Cite this: RSC Adv., 2019, 9, 33789

\title{
A simple and green synthesis of carbon quantum dots from coke for white light-emitting devices
}

\author{
Xiaoting Feng (D)* and Yi Zhang* \\ Coke is a by-product of coal. This paper reports a simple and green chemical oxidation method for carbon \\ quantum dots (CQDs) from coke for use in novel applications. The CQDs emit blue fluorescence and have \\ a fluorescence quantum yield of $9.2 \%$ and blue-green-red spectral composition of $48 \%$. A light-emitting \\ diode (LED) was fabricated by combining the CQDs as a white-light converter with an ultraviolet chip. \\ The Commission Internationale de L'Eclairage chromaticity coordinate $(0.31,0.35)$ and correlated color \\ temperature $(5125 \mathrm{~K})$ of the LED are located in a cool white light zone, suggesting that they have \\ superior potential application in lighting devices.
}

Received 1st September 2019

Accepted 15th October 2019

DOI: $10.1039 / c 9 r a 06946 a$

rsc.li/rsc-advances

Coke is a cheap and abundant by-product obtained from coal

\section{Introduction}

Luminescent carbon quantum dots (CQDs), a new kind of quantum dots (QDs), have received broad attention owing to their superior properties such as variable photoluminescence (PL) behaviour, low toxicity and excellent biocompatibility. ${ }^{\mathbf{1 , 2}}$ They hold great promise in many application fields such as fluorescent probes, ${ }^{3,4}$ fluorescent ink, ${ }^{5}$ bioimaging, ${ }^{6}$ solar cells, ${ }^{7}$ photocatalytic behaviour, ${ }^{8}$ and light-emitting devices. ${ }^{9-11}$

Light-emitting devices (LEDs) are considered a promising substitute for conventional illumination systems owing to their small volume, high energy efficiency, low power consumption and long lifetime. ${ }^{12}$ In general, the most prevalent route for obtaining white LEDs is to combine a blue chip with a yellowemitting phosphor. ${ }^{\mathbf{1 3 , 1 4}}$ However, rare-earth phosphors have obvious drawbacks including high cost and limited resources which restrict further development. ${ }^{15}$ Semiconductor quantum dot phosphors often exhibit aggregation and environmentally unfriendly issues such as containing cadmium or other heavy metals, which largely limits their practical application. ${ }^{\mathbf{1 6 , 1 7}}$ Thus, CQDs with low cost, low toxicity and broad visible light emission tend to become desirable phosphors. Recently, several white LED devices with ultraviolet (UV) chips as excitation light have also been researched. Compared with blue chips, the ultraviolet chip has higher energy to radiate phosphor, which leads to an increased emission intensity of the phosphor.

CQDs have been synthesized from various carboncontaining precursors including small organic carbon sources (e.g. phenylenediamine, ${ }^{18}$ citric acid ${ }^{19,20}$ glucose,${ }^{21}$ L-ascorbic acid, ${ }^{22}$ and thiosalicylic acid. ${ }^{23}$ ). However, the relatively high cost of mentioned carbon precursors above limits the practical applications of CQDs.

Department of Chemistry and Chemical Engineering, Lu Liang University, Lvliang 033001, China.E-mail: xiaotingfeng@llhc.edu.cn; yiyi9900474@163.com products, which has high content of carbon and low content of ash. Coke is mainly used as a combustible energy resource. In recent years, the environmental pollution issues in the coal chemical industry have become very serious. Therefore, it is essential that clean utilization technologies for coal chemical products are developed. There are some works about coal-based CQDs. For example, $\mathrm{Wu}$ et al. prepared photoluminescent CQDs from petroleum coke by ultrasonic-assisted chemical oxidation method. ${ }^{24}$ Petroleum coke was oxidized first in concentrated $\mathrm{H}_{2} \mathrm{SO}_{4}$ and $\mathrm{HNO}_{3}$, and then functionalized by hydrothermal ammonia treatment. The resulting CQDs have excellent water solubility. Hu et al. reported a new strategy to synthesize size-controlled fluorescent CQDs from coal by a combination of carbonization and acidic oxidation etching. ${ }^{25}$ The as-prepared CQDs were used as fluorescence turn-off probe for fast detection of $\mathrm{Cu}^{2+}$ in aqueous solutions. Geng et al. developed a strategy for producing orange fluorescent CQDs from coal tar by a combination of acidic oxidation etching and solvothermal method. ${ }^{26}$ And as-fabricated CQDs exhibit low cytotoxicity and good biocompatibility with cells, making them highly potential for the in vitro and in vivo imaging applications. Although some amazing advances have been made in the applications of the CQDs from coal products, these reported preparation methods of CQDs are multi-step and involve complicated postprocessing processes of removing the excess oxidizing agents. A simple and green synthetic approach and widening the application of coal-based CQDs are still in great demand.

Here we report fluorescent CQDs as a white-light converter for white LED, being prepared by a one-step and green chemical oxidation using low-cost coke as starting materials and hydrogen peroxide as oxidizing agent (Fig. 1). The excess oxidizing agent $\left(\mathrm{H}_{2} \mathrm{O}_{2}\right)$ was removed through solution boiling without addition of bases to neutralize. The obtained CQDs are nano-sized and emit blue fluorescence. The emission spectrum of the CQDs exhibits a broad emission covering entire visible 


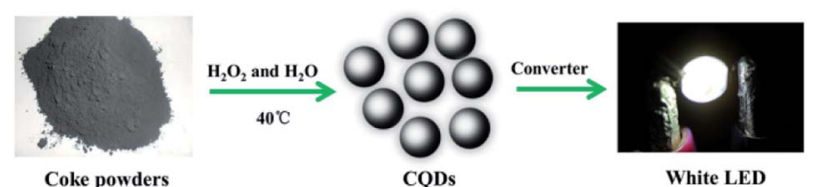

Fig. 1 Synthesis scheme of CQDs from coke and their applications in white LED

region with high blue-green-red spectral composition, which contributes to single phosphors to form white light. Ultimately, a cool white LED was constructed by combining the coke-based CQDs and UV-LED chip. The optoelectronic device application of the coke-based CQDs is demonstrated.

\section{Experimental}

\subsection{Materials}

Coke powders were obtained from Shanxi Datuhe Coking Co., Ltd (Shanxi, China). Hydrogen peroxide (30\% wt\%) was purchased from Kaitong Chemical Reagent Factory (Tianjin, China). Acetic acid (36\% wt $\%)$ was acquired from Guangfu Technology Development (Tianjin, China). Dialysis bag (molecular weight cut-off 1000) was supplied by Union Carbide Corporation (USA). UV-LED chip ( $365 \mathrm{~nm}, 1.0 \mathrm{~W}$ ) was provided from Guanghuashi Technology Co., Ltd (Shenzhen, China).

\subsection{Synthesis of CQDs}

Coke powders ( $300 \mathrm{mg}$ ) were put into a mixture of $\mathrm{H}_{2} \mathrm{O}_{2}(20 \mathrm{~mL})$ and $\mathrm{H}_{2} \mathrm{O}(30 \mathrm{~mL})$ in a beaker under ultrasonic conditions for $0.5 \mathrm{~h}$. The obtained mixture was heated at $40{ }^{\circ} \mathrm{C}$ under vigorous stirring conditions for $3 \mathrm{~h}$. After the reaction, the solution was heated to the boiling point to remove the excess $\mathrm{H}_{2} \mathrm{O}_{2}$. The resulting solution was filtered using $0.22 \mu \mathrm{m}$ polyether sulfone membranes and then purified for $12 \mathrm{~h}$ in a dialysis bag (Da 1000) to remove some the larger nanoparticles or impurities. The purified solution was kept for characterization such as UV absorption, photoluminescence behavior, and quantum yield measurements. Then, the purified CQDs solution was freezedried in a vacuum freeze dryer. The powdered CQDs were obtained and collected for characterization such as morphology, surface structure, and device performance.

\subsection{Device fabrication}

The CQDs as a white light converter were applied to fabricate white LED devices. A few drops of CQDs/epoxy resin aquogel were dispensed into an optical lens, followed by leaving the optical lens in a vacuum chamber at $80{ }^{\circ} \mathrm{C}$ for $6 \mathrm{~h}$ to remove bubbles. Finally, the optical lens was solidly placed on the bottom of the UV chip with the emission wavelength centered at $365 \mathrm{~nm}$.

\subsection{Characterization}

The morphology of the CQDs was examined by transmission electron microscopy (TEM) on a JEOL JEM-2010 at an accelerating voltage of $200 \mathrm{kV}$. The surface structure of the CQDs was characterized by Fourier transform infrared (FT-IR) spectroscopy. FT-IR spectrum were recorded on a BRUKER TENSOR 27 spectrometer in the form of KBr pellets. The optical properties of the CQDs were measured on an Ultraviolet-visible (UV-vis) spectrophotometer and a luminescence spectrometer. UV-vis absorption spectrum of CQDs was obtained by a Hitachi U3900 UV-vis spectrophotometer. The PL spectra were recorded on a Horiba Fluoromax-4 luminescence spectrometer with a Xe lamp as the excitation source. The Spectra Scan PR655 was applied to analyze the correlated color temperature (CCT), emission spectra and Commission Internationale de L'Eclairage (CIE) chromaticity coordinate of the LED.

\subsection{Quantum yield (QY) measurements}

The fluorescence QY of CQDs was determined according to an established procedure using a comparative method. ${ }^{27}$ Quinine sulfate (literature $\mathrm{QY}=0.54$ ) in $0.10 \mathrm{M} \mathrm{H}_{2} \mathrm{SO}_{4}$ was selected as the reference sample to determine the QY of CQDs solutions at different concentrations. All the optical absorbance values of the solutions were recorded at $360 \mathrm{~nm}$ excitation, which should not exceed 0.1 in order to minimize internal filter effects. The integrated fluorescence intensity is calculated according to the area under the photoluminescence curve from 380 to $700 \mathrm{~nm}$. The graphs were plotted using integrated fluorescence intensity versus the absorbance. The QY of CQDs was calculated by the following equation:

$$
Q_{\mathrm{x}}=Q_{\mathrm{s}}\left(k_{\mathrm{x}} / k_{\mathrm{s}}\right)\left(\eta_{\mathrm{x}} / \eta_{\mathrm{s}}\right)^{2}
$$

where $Q$ is quantum yield, $k$ is the slope of fitted line, and $\eta$ is refractive index of the solvent. The subscripts "s" and " $\mathrm{x}$ " refer to the quinine sulfate and CQDs, respectively. For the solutions in the present study, $\eta_{\mathrm{x}} / \eta_{\mathrm{s}}=1$.

\section{Results and discussion}

The morphology of CQDs was investigated using TEM measurements. As shown in Fig. 2(a), the CQDs are spherical and well dispersed without apparent aggregation. The average particle size of CQDs was calculated to be $6.5 \mathrm{~nm}$. To further investigate the chemical structure of CQDs, an FT-IR spectrum was obtained, as shown in Fig. 2(b). The broad characteristic band at $3415 \mathrm{~cm}^{-1}$ is related to the stretching vibration of $\mathrm{O}-\mathrm{H}$. The band at $1564 \mathrm{~cm}^{-1}$ originates from the stretching vibration of $\mathrm{C}=\mathrm{O}$ in the carboxylic groups. The band at $1681 \mathrm{~cm}^{-1}$ corresponds to the stretching vibration of $\mathrm{C}=\mathrm{C}$. The CQDs exhibited several characteristic absorption bands of $\mathrm{C}-\mathrm{H}$ $\left(2960 \mathrm{~cm}^{-1}\right)$ and C-O-C $\left(1267 \mathrm{~cm}^{-1}\right)$. It can be speculated that massive hydroxyl groups are situated on the surface of the CQDs.

UV-vis absorption and PL spectra were investigated to explore the optical properties of the CQDs. From the UV-vis absorption spectrum (Fig. 3(a)), the peak at $289 \mathrm{~nm}$ is ascribed to the $\mathrm{n} \rightarrow \pi^{*}$ transition of $\mathrm{C}=\mathrm{O}$, which is corresponding to the previously reported absorption peak at 279$355 \mathrm{~nm}$ ascribed to $\mathrm{n} \rightarrow \pi^{*}$ of $\mathrm{C}=0 .{ }^{28,29}$ In Fig. 3(b), fluorescent 

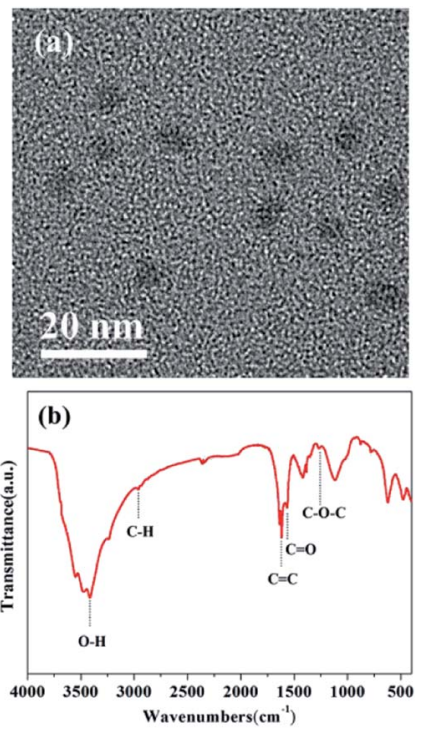

Fig. 2 (a) TEM image of CQDs. (b) FT-IR spectrum of CQDs.

measurements were carried out with different excitation wavelengths. As the excitation wavelength changes from 310 to $420 \mathrm{~nm}$, PL emission intensity of CQDs aqueous solutions first increases in the $310-330 \mathrm{~nm}$ range and then gradually decreases in 330-420 $\mathrm{nm}$ range. When excited at $330 \mathrm{~nm}$, the CQDs exhibit maximum PL emission. The maximum emission wavelength is located at $410 \mathrm{~nm}$. When excited at $310 \mathrm{~nm}$, the fluorescence intensity of CQDs reaches its minimum at $403 \mathrm{~nm}$. The emission peak of CQDs shows red-shift with the increase in excitation wavelength. According to above-mentioned situation, the CQDs exhibit excitation-wavelength-dependent PL emission, which is contributed to different energy level structures of the CQDs. ${ }^{30,31}$ Surface states are known to induce energy level
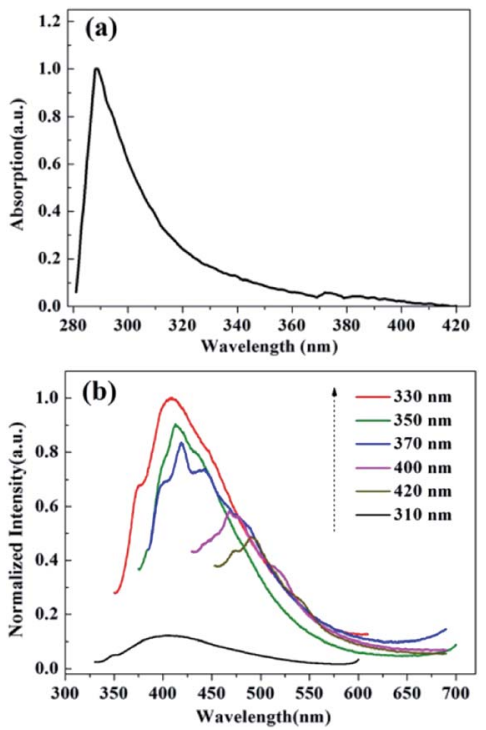

Fig. 3 (a) UV-vis absorption spectrum of CQDs. (b) PL spectra of CQDs at different excitation wavelengths. structures. The multiple energy levels in the different surface states of CQDs result in excitation-wavelength-PL property. ${ }^{32}$

In Fig. 4(a), the CQDs aqueous solutions show a strong peak at $422 \mathrm{~nm}$ and broad visible light emission when excited at $365 \mathrm{~nm}$. Deionized water showed no fluorescence a $365 \mathrm{~nm}$ UV lamp (inset, left, in Fig. 4(a)), while the CQDs aqueous solution emitted bright blue luminescence under UV light (inset, right, in Fig. 4(a)). In Fig. 4(b), then the PL spectrum under excitation of $365 \mathrm{~nm}$ is divided into six colors (violet for $380-450 \mathrm{~nm}$, blue for 450-495 nm, green for 495-570 nm, yellow for 570-590 nm, orange for $590-650 \mathrm{~nm}$, red for $650-700 \mathrm{~nm}$ ) to measure the spectral composition of the CQDs. The spectral composition of CQDs indicates the proportion of blue-green-red spectrum can reach a rather high index of $48 \%$, which is higher than the bluegreen-red proportion value of $44 \%$ previously researched. ${ }^{33} \mathrm{~A}$ high blue-green-red spectral composition is conducive to a promotion in the efficiency of CQDs as a single white-light converter for white LED with low CCT. This phenomenon may be related to the $\pi$-electron density on the surface of CQDs. The energy gaps of the CQDs might decrease due to the existence of high $\pi$-electron density, consequently resulting in a redshift emission. As a result of this, the blue-green-red spectral composition of CQDs may increase. ${ }^{34,35}$

The QY of the CQDs is measured with quinine sulfate as a standard $(\mathrm{QY}=54 \%) .{ }^{27}$ The relative QY of the coke-based CQDs is calculated to be $9.2 \%$ (Fig. 5), comparable to QY

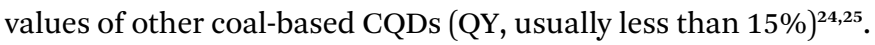
Some researchers consider QY are dependent on the probability of electron-hole radiative recombination from the excited state to ground state. ${ }^{36}$ There remains broad research space for QY of coke-based CQDs.

To explore the potential of the coke-based CQDs which have exhibited excellent optical properties, we have fabricated a white LED with UV chip as excitation light and the obtained coke-based CQDs as a white light converter. Fig. 6(a) shows
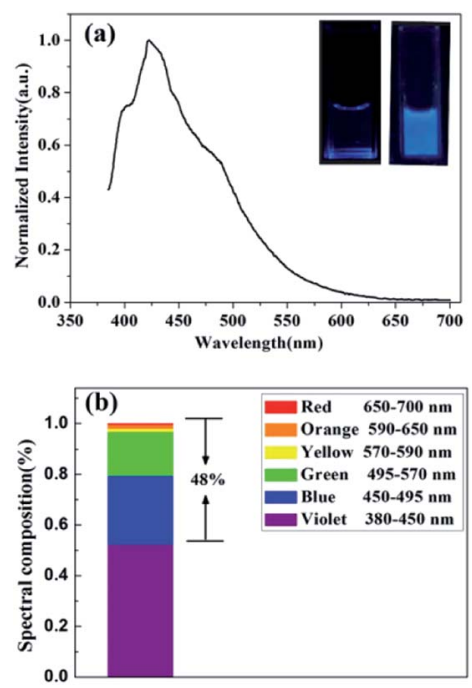

Fig. 4 (a) PL spectrum of CQDs under $365 \mathrm{~nm}$, inset: photographs of deionized water (left) and CQDs in deionized water (right) under $365 \mathrm{~nm}$ UV light. (b) Spectral composition of CQDs. 


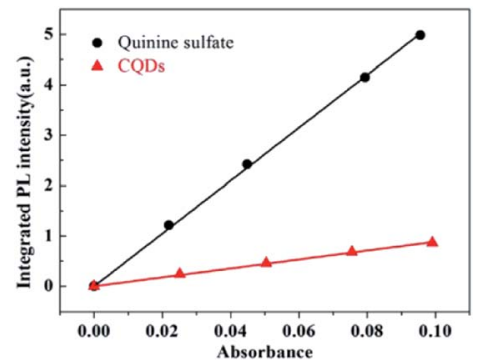

Fig. 5 QY measurement of CQDs.

a cross-section view of the LED based on coke-based CQDs. It can be seen that the LED is basically fabricated with gold wires, electrodes, a lens and UV chip. Coke-based CQDs as a light converter are coated onto the inner wall of the lens. The ends of the chip are connected to anode and cathode of the power supply, respectively. CQDs are excited by the UV chip and then emit broad visible light, which can convert the ultraviolet light into white light. In addition, the optical stability of the LED based on the CQDs was investigated at different time intervals in Fig. 6(b). The emission peak of the LED exhibits no apparent shift and the emission intensity decreases slightly after continuous illumination for $4 \mathrm{~h}$ at $3.2 \mathrm{~V}$, which indicate the excellent stability of the device.

As shown in Fig. 7(a), the emission spectrum of the LED is broad and is spread over almost the entire visible range (400$720 \mathrm{~nm}$ ). The as-prepared LED emits bright white light (inset in Fig. 7(a)). White light is made up of blue-green-red spectrum of the CQDs. The emission peak before $400 \mathrm{~nm}$ originates from the UV chip and is not involved in the formation of white light, which has no effect on the CIE coordinates of the device. ${ }^{37}$ Apart from the emission at $380 \mathrm{~nm}$ directly from the UV chip, the
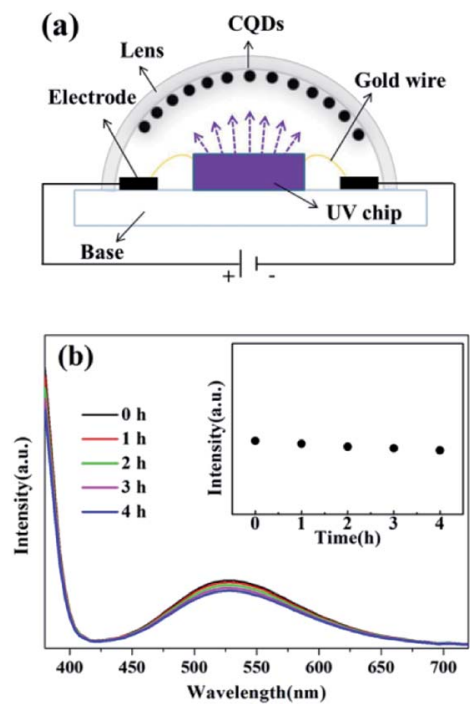

Fig. 6 (a) A cross-section view of the LED based on coke-based CQDs. (b) Emission spectra of the white LED at different working time intervals operated at $3.2 \mathrm{~V}$. Inset: Emission intensity with different working time intervals.
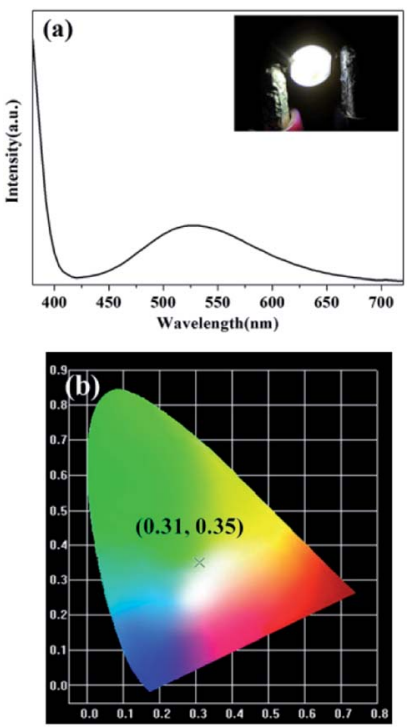

Fig. 7 (a) Emission spectrum of the white LED, inset: the photograph of the LED operated at $3.2 \mathrm{~V}$. (b) CIE 1931 chromaticity diagram of the white LED.

emission spectrum centered at $528 \mathrm{~nm}$ originates from the CQDs. The maximum emission wavelength of the LED shifts to longer wavelength compared with that of the CQDs aqueous solutions. This observation might be contributed to the energy transfer caused by light reabsorption of solid state CQDs. ${ }^{\mathbf{1 0 , 1 2}}$ Shorter-wavelength light with high-energy emitted from CQDs might be partly reabsorbed by the surrounding CQDs, from which longer-wavelength light is emitted. In Fig. 7(b), the CIE chromaticity coordinate of the device is located at $(0.31,0.35)$. The correlated color temperature is $5125 \mathrm{~K}$, corresponding to a cool white light gamut and being suitable for outdoor lighting.

\section{Conclusions}

In summary, we have demonstrated a green and simple method to prepare fluorescent CQDs from coke for white light-emitting devices. The prepared CQDs, with quantum yield of $9.2 \%$ and blue-green-red spectral composition up to $48 \%$, were synthesized via a one-step chemical oxidation from coke as starting materials and hydrogen peroxide as oxidizing agent. The structural characterization of CQDs by FT-IR analysis demonstrates the CQDs contain carboxyl group and other O-rich groups. The obtained CQDs emit blue fluorescence under UV light illumination and exhibit excitation-dependent PL emission. By taking advantage of these remarkable properties, the CQDs based white LED exhibits bright cool-white light with a CIE chromaticity coordinate of $(0.31,0.35)$ and a CCT of 5125 K. This study demonstrates that the coke-based CQDs as candidate phosphors have promising potential applications in light-emitting devices.

\section{Conflicts of interest}

There are no conflicts to declare. 


\section{Acknowledgements}

This work was supported by Shanxi Province Science Foundation for Youths (201801D221110, 201801D221106), Technological Innovation Foundation of the Higher Education Institutions of Shanxi Province (2019L0950, 2019L0946) and the Key Research Program of Lvliang City (No. 201701103, No. 201701102).

\section{Notes and references}

1 J. Zhang, X. An, X. Li, X. Liao, Y. Nie and Z. Fan, Chem. Phys. Lett., 2018, 706, 702-707.

2 G. Lecroy, P. Wang, C. Bunker, K. Fernando, W. Liang, L. Ge, M. Reibold and Y. Sun, Chem. Phys. Lett., 2019, 724, 8-12.

3 Y. Li, Y. Liu, X. Shang, D. Chao, L. Zhou and H. Zhang, Chem. Phys. Lett., 2018, 705, 1-6.

4 Y. Zhang, P. Cui, F. Zhang, X. Feng, Y. Wang, Y. Yang and X. Liu, Talanta, 2016, 152, 288-300.

5 J. Wang, C. Wang and S. Chen, Angew. Chem., Int. Ed., 2012, 51, 9297-9301.

6 H. Ding, S. Yu, J. Wei and H. Xiong, ACS Nano, 2016, 10, 484491.

7 C. Liu, K. Chang, W. Guo, H. Li, L. Shen, W. Chen and D. Yan, Appl. Phys. Lett., 2014, 105, 073306.

8 S. Hu, Z. Wei, Q. Chang, A. Trinchi and J. Yang, Appl. Surf. Sci., 2016, 378, 402-407.

9 Y. Miao, K. Wang, B. Zhao, L. Gao, P. Tao, X. Liu, Y. Hao, H. Wang, B. Xu and F. Zhu, Nanophotonics, 2018, 7, 295-304. 10 J. Wang, F. Zhang, Y. Wang, Y. Yang and X. Liu, Carbon, 2018, 126, 426-436.

11 J. Zheng, Y. Wang, F. Zhang, Y. Yang, X. Liu, K. Guo, H. Wang and B. Xu, J. Mater. Chem. C, 2017, 5, 8105-8111.

12 Y. Miao, X. Wei, L. Gao, K. Wang, B. Zhao, Z. Wang, B. Zhao, H. Wang, Y. Wu and B. Xu, Nanophotonics, 2019, 8, 17831794.

13 M. Shang, C. Li and J. Lin, Chem. Soc. Rev., 2014, 43, 13721386.

14 Q. Zhang, C. Wang, L. Ling and S. Chen, J. Mater. Chem. C, 2014, 2, 4358-4373.

15 Q. Chen, C. Wang and S. Chen, J. Mater. Sci., 2013, 48, 23522357.

16 P. Tao, Y. Miao, H. Wang, B. Xu and Q. Zhao, Chem. Rec., 2019, 19, 1531-1561.
17 Y. Miao, K. Wang, L. Gao, B. Zhao, H. Wang, F. Zhu, B. Xu and D. Ma, J. Mater. Chem. C, 2018, 6, 8122-8134.

18 K. Jiang, S. Sun, L. Zhang, Y. Lu, A. Wu, C. Cai and H. Lin, Angew. Chem., Int. Ed., 2015, 54, 5360-5363.

19 J. Xu, Y. Miao, J. Zheng, H. Wang, Y. Yang and X. Liu, Nanoscale, 2018, 10, 11211-11221.

20 J. Xu, Y. Miao, J. Zheng, H. Yang and X. Liu, Adv. Opt. Mater., 2018, 6, 1800181.

21 X. Feng, F. Zhang, Y. Wang, Y. Zhang, Y. Yang and X. Liu, J. Electron. Mater., 2016, 45, 2784-2788.

22 X. Feng, Y. Zhao, L. Yan, Y. Zhang, Y. He, Y. Yang and X. Liu, J. Electron. Mater., 2015, 44, 3436-3443.

23 X. Feng, F. Zhang, Y. Wang, Y. Zhang, Y. Yang and X. Liu, Appl. Phys. Lett., 2015, 107, 213102.

24 M. Wu, Y. Wang, W. Wu, C. Hu, X. Wang, J. Zheng, Z. Li, B. Jiang and J. Qiu, Carbon, 2014, 78, 480-489.

25 C. Hu, C. Yu, M. Li, X. Wang, J. Yang and Z. Zhao, Small, 2014, 10, 4926-4933.

26 B. Geng, D. Yang, F. Zheng, C. Zhang, J. Zhan, Z. Li, D. Pan and L. Wang, New J. Chem., 2017, 41, 14444-14451.

27 Y. Zhang, Y. Wang, X. Feng, F. Zhang, Y. Yang and X. Liu, Appl. Surf. Sci., 2016, 387, 1236-1246.

28 H. Nie, M. Li, Q. Li, S. Liang, Y. Tan, L. Sheng, W. Shi and S. Zhang, Chem. Mater., 2014, 26, 3104-3112.

29 L. H. Zhu, W. Zhang and S. Yu, Nanoscale, 2013, 5, 17971802.

30 Y. Cui, Z. Hu, C. Zhang and X. Liu, J. Mater. Chem. B, 2014, 2, 6947-6952.

31 S. Hu, Y. Wang, W. Zhang, Q. Chang and J. Yang, Materials, 2017, 10, 165.

32 X. Li, Y. Liu, X. Song, H. Wang, H. Gu and H. Zeng, Angew. Chem., Int. Ed., 2015, 54, 1759-1764.

33 W. Kwon, S. Do, J. Lee, S. Hwang, J. Kim and S. Rhee, Chem. Mater., 2013, 25, 1893-1899.

34 Y. Wang, J. Zheng, J. Wang, Y. Yang and X. Liu, Opt. Mater., 2017, 73, 319-329.

35 L. Bao, C. Liu, Z. Zhang and D. Pang, Adv. Mater., 2015, 27, 1663-1667.

36 F. Zhang, Y. Wang, Y. Miao, Y. He, Y. Yang and X. Liu, Appl. Phys. Lett., 2016, 109, 083103.

37 F. Zhang, X. Feng, Y. Zhang, L. Yan, Y. Yang and X. Liu, Nanoscale, 2016, 8, 8618-8632. 